\title{
Assessment of teaching and learning by mixed diagnostic testing
}

\author{
Anna Yankovskaya ${ }^{1}$, Ilya Levin ${ }^{2}$ and Irina Fuks 3 \\ ${ }^{1}$ Computer Science Department, Tomsk State University, Tomsk, Russia \\ Intelligent Systems Laboratory, Tomsk State University of Architecture and Building, Tomsk, Russia \\ Computer Systems Department, Tomsk State University of Control Systems and Radioelectronics, Tomsk, Russia \\ 2 Department of Math, Science and Technology Education, Tel Aviv University, Tel Aviv, Israel \\ ${ }^{3}$ Computer Science Department, Tomsk State University, Tomsk; Russia \\ For correspondence: ilia1@post.tau.ac.il
}

\begin{abstract}
The paper deals with assessment of educational process. Specifically, monitoring and testing of students' knowledge, of professional and personal skills/abilities are in the focus of the study. A new assessment approach called Mixed Diagnostic Testing (MDT) is discussed in the paper. The approach combines two known testing concepts: unconditional testing and conditional testing. In the unconditional testing, each next question of the test sequence is independent of previous test results; in contrast with it the conditional testing includes a sequence of questions strongly connected with the previous test results. The proposed approach provides designing of an individual learning "trajectory" for each student. According to our hypothesis, such a trajectory is especially important since it allows personalizing the learning process, thus increasing its efficiency. The paper provides theoretical analysis of the MDT approach, as well as of its implementation in a certain academic course "Information technologies". For preparing (constructing) of mixed diagnostic tests, experts' knowledge of specific subjects should be utilized. It is shown, that the use of MDT effectively supports and facilitates development of curriculum. The MDT reduces both time and cost expenses for organization and management of the educational process. Since the MDT may actually replace a teacher in the teacher's function as a consultant, the proposed approach is especially promising in the blended learning.
\end{abstract}

Keywords: blended learning, information technology, learning trajectory, assessment, mixed diagnostic test.

\section{Introduction}

Emerging information technologies provide a number of innovative and very promising technologies for teaching and learning. A significant group of the technologies are related to the distance, on-line education or to the education involving limited interaction between students and teachers. During the last five years, so-called Web 2.0 technologies have become ubiquitous (Levin \& Kojukhov, 2013). Such technologies affect both the educational practice and the principle approaches to the distance education by improving collaboration which is a very important component of education. At the same time, the problem of evaluation and improving efficiency of the educational process becomes actual even more than in a conventional class, due to absence of the teacher in such a class.

The concept of blended learning, which is a learning combining both education and training, was introduced in (Bliuk et al, 2007). Being a new theoretical concept, at the same time the blended learning is a popular approach both in a higher education and in an industrial training.

In general, the blended learning corresponds to an integrated learning environment, which combines e-learning with traditional classroom teaching (Graham, 2006). One of the main problems of high school is to develop mechanisms for effective training a large number of students by a wide diversity of tasks (Singera and Stoicescu, 2011).

The simplest example of the blended learning is using diverse resources and methods within the structured and targeted syllabus. Despite of the variety of information technologies (including methods of artificial intelligence), methods for diagnostics of the gained knowledge in the teacherstudent interaction are not developed enough. The development of such methods is highly time- 
consuming and requires considerable efforts (Brusilovsky et al, 2006, Uskov and Uskov, 2010).

Students of different abilities have diverse preferences in learning and achieving goals of lessons. Ausburn (2004) used a questionnaire aimed to study students' abilities, such as the initial level of their knowledge, skills, and experience. Orientation on student's particular interests and capacities should make the learning process more effective. Any graduate student (a specialist) should rely on experience and skills obtained at a higher school to find the proper solution of educational and industrial tasks. Moreover, the specialist should understand very clearly why and how he/she will use the obtained knowledge, professional and personal skills, and abilities to reach his/her goals. Bliuk et al (2007) identify four different ways to define the blended learning. The blended learning can be considered as: a) combining modes of web-based technology; b) combining of various pedagogical approaches; c) combination of any forms of instructional technology with a face-to-face instructor-led training; or d) combination of teaching with practical work.

In our paper, we propose a new approach for training and testing. The approach is based on so-called mixed diagnostic tests (MDT) introduced in (Yankovskaya et al, 1996, 1997, 2009, 2010, 2011). The MDT uses both conditional and unconditional components of training.

The blended learning is known as the learning with a high level of redundancy of possible learning trajectories. An important example of the blended learning with high level of redundancy is learning subjects owing multiple representations (Levin and Levit, 1998; Levin et al, 2004). In (Yankovskaya et al, 2013) the MDT is used in order to design specific trajectories of students' educational processes.

For effective implementation of the MDT approach, a number of tasks have to be solved: a) providing students with an instrument for designing their own learning trajectories; b) providing interactions teacher-student and student-student; and c) increasing accessibility of information.

The main idea of the present study is to make the MDT process transparent for students studying the same topic. It provides the students with a possibility to collaborate not just with the teacher but also with one another, comparing their own results and achievements.

\section{Problem Background}

The modern society involves dynamic, frequently unpredictable changes, which call for students and graduates to be able to solve different educational and professional tasks (Singera and Stoicescu, 2011). It should be noted that the blended learning requires broadening of the range of skills, experience and competence both of teachers and students.

In (Yankovskaya, 1996), the mixed diagnostic test was proposed as a new approach of intelligent systems design. The approach is based on pattern recognition methods. Implementation of the MDT in intelligent learning and training systems was presented in (Yankovskaya, 2011), (Yankovskaya and Semenov, 2013).

Unfortunately, presently available testing methods motivate students very insufficiently. The main advantage of the MDT approach is in providing flexibility in designing of learning trajectories. According to our hypothesis, more flexible leaning trajectories allow increasing motivation of students and improving quality of the learning process.

Any academic discipline may be represented as a structure comprising a sequence of content blocks. In order to formalize description of such a structure, it is accepted to associate each of the content blocks with a specific didactic unit. A sequence of didactic units can be either arbitrary, or be dependent on the students' abilities expressed by their success in studying previous didactic units.

In turn, for each student, the study of individual didactic units can be represented as the sequence of independent content blocks of the course. The MDT can be applied for development of separate didactic units. 


\section{Basic Concepts and Definitions}

In our paper, the following main concepts are used (Yankovskaya, 2011), (Yankovskaya and Semenov, 2013):

Respondent - a person participating in a testing process.

Diagnostic test - a set of test tasks, that is able to identify such material of the course, on which the respondent has failed.

Mixed diagnostic test - a diagnostic test comprising a sequence of test tasks built on the base of results of performing previous tasks by the respondent.

Unconditional portion of (diagnostic) test (UCT) is a portion of the test that includes tasks being independent from previous test results. UCT allows a concurrent representation of its constituent tasks.

Conditional portion of (diagnostic) test (CCT) is a portion of the test that includes tasks, sequence of which depends on previous test results. The CCT is characterized by the sequential representation of test tasks.

MDT tree - a structure representing relationships between separate blocks (tasks) of the MDT. The root node is associated with the unconditional component of MDT. Each of the remaining nodes is associated with either unconditional or conditional component of MDT. The MDT tree edges define connections between nodes (components of the MDT).

\section{The Problem}

Developing the mixed diagnostic tests (MDT) is in the focus of our research. Additionally, we study the way of MDT implementation by using some specific didactic unit of the course "Information Technology".

The course "Information Technology" of the Computer Engineering BSc curriculum is used in our study. The MDT for the course has been developed by a group of highly qualified Information Technology experts.

\section{Description of the Test Structure}

Traditionally, the study of the discipline "Information Technology" is assessed by a grading test. Presently, the test uses Moodle in majority of universities. Some of the Moodle test tasks were used for developing the MDT. The tasks were proposed to the students of the Tomsk State University. Each test task includes a question and a certain set of possible answers. In a number of tasks, students were asked to formulate the correct answer themselves. In our study, four standard forms of test tasks were used. These forms are:
a) a closed form with the only answer,
b) a closed form with multiple answers,
c) a matching test,
d) a sequential test.

The test consists of 25 questions. Each of the questions is chosen randomly from a certain category of questions. Results of the test were assessed by a five-point grading scale.

Results of a statistical analysis of the test tasks provided by the Moodle are shown in Fig. 1.

Statistical analysis of the test indicates that some test tasks have low discriminative efficiency for tracking of the knowledge perceiving. However, they may be useful for managing the educational 
process, since their facility index indicates the level of complexity of the tasks.

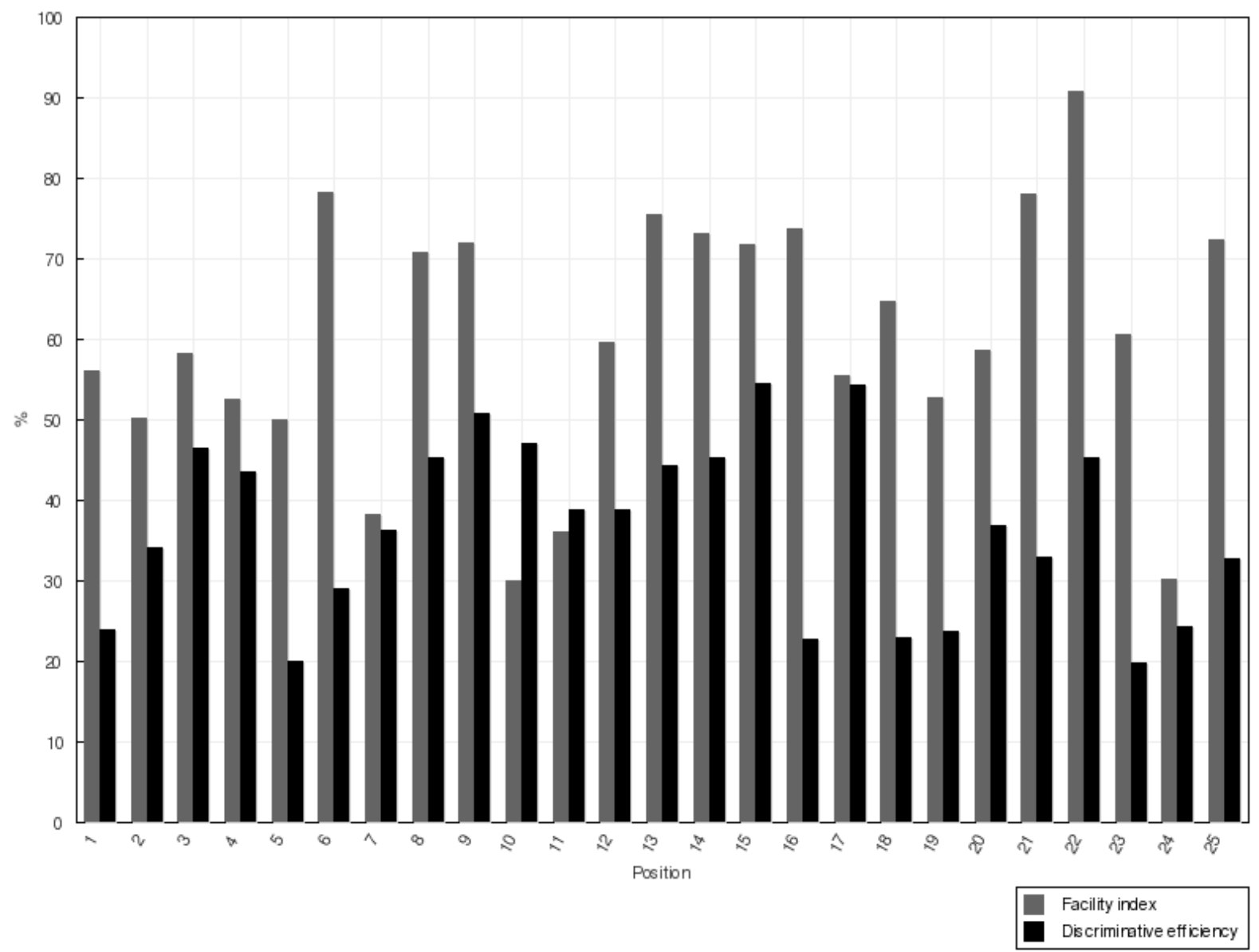

Figure 1. Statistics for question positions

An MDT tree created for a specific topic "processing of text by using Word" is shown in Fig. 2. The study of the topic should form a system of knowledge about the principles of working with electronic text documents and the basics of personal information management. All nodes of the tree are numbered. Symbol $u_{i}$ is associated with the unconditional components, $c_{i}$ - with the conditional one. Table 1 shows the correspondence between the test tasks and the MDT.

\begin{tabular}{|c|c|}
\hline $\begin{array}{l}\text { Test question } \\
\text { number }\end{array}$ & The questions in the MDT \\
\hline 6 & Paragraph - Attributes \\
\hline 7 & Symbol - Attributes \\
\hline 8 & Section, List, Paragraph - Attributes \\
\hline 18 & Style, Standard styles, Standard Table of Contents \\
\hline 19 & Form, Template, Reference \\
\hline 23 & Bibliography, Reference to a Bibliography \\
\hline 24 & Paragraph - Attributes \\
\hline
\end{tabular}

In the test tree shown in Figure 2, five unconditional components are highlighted. Both the learning and the testing start from the first unconditional component "Formatting". The next level of the test comprises 4 unconditional components: "Symbol", "Paragraph", "Special Parts of the Document", "Automatic Document". On this level, any of the unconditional components can be selected. The 
average assessment is followed by fulfillment of tasks of the unconditional portion of the test.

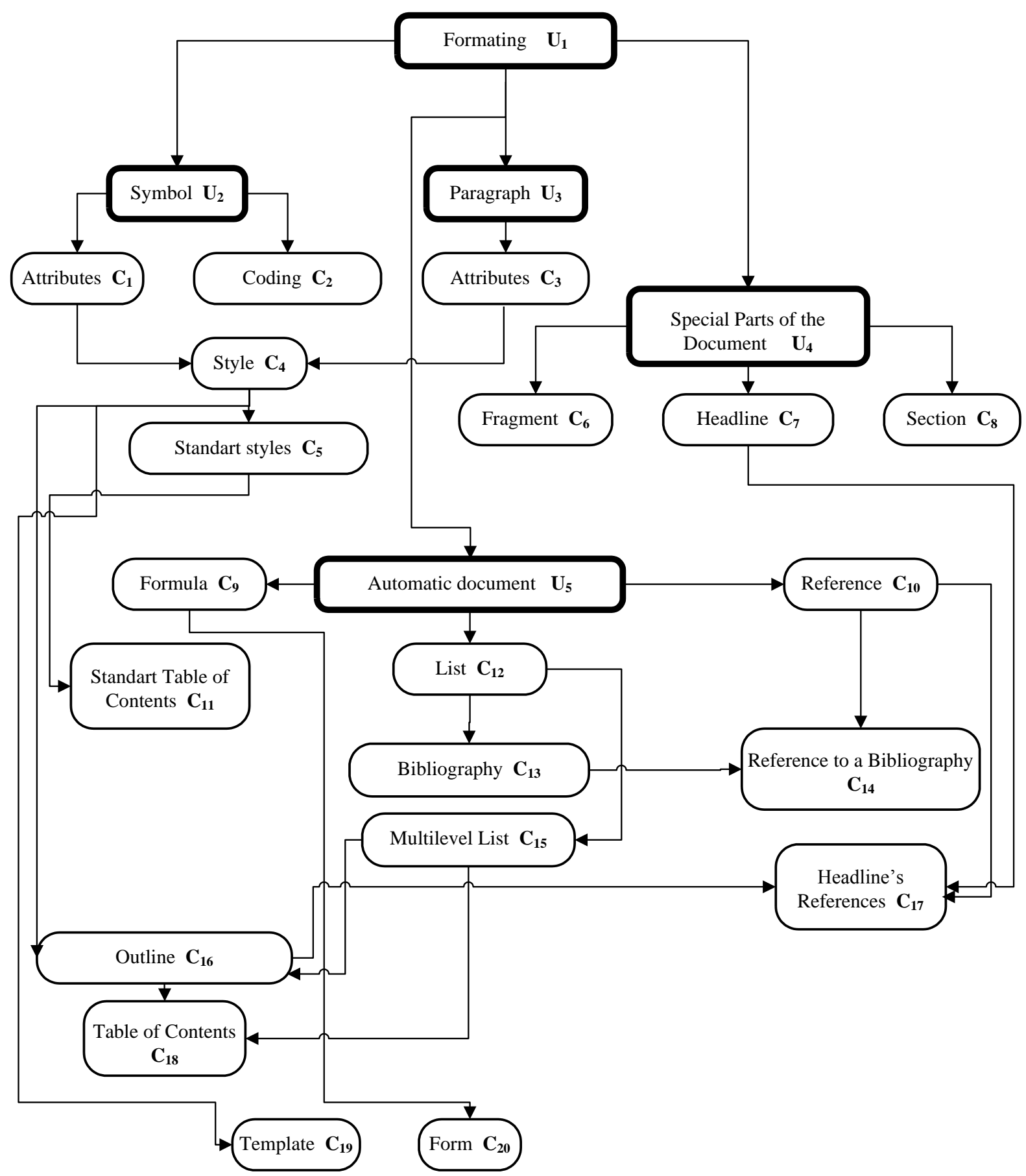

Figure 2. Mixed diagnostic test tree

The further testing is implemented on the base on the above assessment. The further testing direction starts from the certain selected unconditional component to the conditional components associated with this component. At each successive stage of the testing, just one test task is proposed. The task is assessed, and then the testing propagates on the selected branch of the test tree. In the case of failure on a certain task, the respondent is redirected to the relevant section of the course for additional studies. If the respondent is still unable to solve this problem alone, he/she is given an opportunity to ask a teacher.

There are three nodes that include a number of input edges. For example, "Outline" and "Headline's 
References". Tasks corresponding to such nodes can be performed only after passing all the higher branches of the tree.

The above strategy of passing the test allows creating the final assessment as an integrated one that includes results gained at each level of the test. Notice that the described procedure also provides a possibility to study the learning and testing processes for further cognitive analysis of students' achievements.

In the next section, we describe an algorithm for implementing the proposed approach. For simplicity, we use the same numbering of the MDT tree nodes as shown in Fig. 2.

\section{Construction of MDT tree Data Structures}

For each unconditional and conditional test components, a so-called coefficients matrix of the correct answers has to be constructed. The matrix comprises weights of the questions (Table 2) (Yankovskaya and Semenov, 2013).

Table 2. Coefficients matrix

\begin{tabular}{|c|c|c|c|c|c|c|c|}
\hline & 1 & 2 & $\ldots$ & $j$ & $\ldots$ & $k$ & weight \\
\hline 1 & $a_{11}$ & $a_{11}$ & $\ldots$ & $\ldots$ & $\ldots$ & $a_{11}$ & $w_{1}$ \\
\hline 2 & $a_{11}$ & $a_{11}$ & $\ldots$ & $\ldots$ & $\ldots$ & $a_{11}$ & $w_{2}$ \\
\hline$\ldots$ & $\ldots$ & $\ldots$ & $\ldots$ & $\ldots$ & $\ldots$ & $\ldots$ & $\ldots$ \\
\hline$i$ & $a_{i 1}$ & $a_{i 2}$ & $\ldots$ & $a_{i j}$ & $\ldots$ & $a_{i k}$ & $w_{i}$ \\
\hline$\ldots$ & $\ldots$ & $\ldots$ & $\ldots$ & $\ldots$ & $\ldots$ & $\ldots$ & $\ldots$ \\
\hline$n$ & $a_{n 1}$ & $a_{n 2}$ & $\ldots$ & $a_{n j}$ & $\ldots$ & $a_{n k}$ & $w_{n}$ \\
\hline
\end{tabular}

Rows of the matrix are associated with the test tasks proposed to a respondent while passing a test component, the columns - the index (number) of respondent's answers, $n$ - the number of questions, $k \geq 2$ - the maximum number of possible answers (both correct and incorrect answers). Answer's weight $w_{i}$ has to satisfy the condition $0<w_{i} \leq 1$ for any $i$.

For test tasks of so-called closed type, with the only possible answer, $k$ is equal to the amount of the proposed answers. For test tasks of the closed type with multiple answers: $k=\sum_{i=1}^{m} C_{m}^{i}$, where $m-$ the number of answers. For example, for the test tasks like comparing two lists: $k=l$, where $l$ - the length of the list. For the test tasks like recognition of the correct sequence: $k=s$ !, where $s-$ the number the sequence elements.

A matrix element at the intersection of the $i$-th row and the $j$-th column specifies weight of the $j$-th answer to the $i$-th question. It should be noted that the total number of answers to different questions may be different, and, as a result, the matrix may contain empty elements.

In addition to the above-described matrix, a so-called threshold assessment of learning material $p_{t}$, where $t$ - the number of MDT tree node, has to be specified.

\section{Test Strategy with Saving Results}

Let the testing always starts at the node $u_{1}$. The respondent is given all the tasks of the test. The respondent's answers are registered as a pair $i, j(i)$, where $i$ - the number of the question, $j(i)$ - the number of the selected answer to the $i$-th question. This information allows reconstructing the process of passing the test by each student. According to the test results, an intermediary assessment 
is calculated $E_{u_{1}}=\left(\sum_{i=1}^{n} a_{i, j(i)} \times w_{i}\right) / n$.

The assessment is compared with a threshold value $p_{u_{i}}$, by using methods of fuzzy logic and threshold logic (Varshavsky, et all, 2003). After that, any other unconditional test component is performed. This component is determined according to the MDT tree. It is obvious that sometimes an additional study of the relevant part is required (Yankovskaya, 2011), (Yankovskaya and Semenov, 2011, 2013).

Similarly, every other component of the unconditional test is tested. Some differences have to be mentioned for cases when the node with a conditional component is reached.

The concept of MDT assumes that only one question is selected from the conventional components of a test. The corresponding answer determines the direction of a so-called tree pass. The respondent's answer is registered as a pair $i, j(i)$, where $i$ - the question number, $j(i)$ - the number of the chosen answer. On the base of the test results, the intermediary assessment is calculated $E_{c_{q}}=a_{i, j(i)} \times w_{i}$. It is compared with the corresponding threshold value $p_{c_{q}}$, where $q$ is the number of a chosen conditional component. After that, another component is performed. This component is determined according to the MDT tree. Sometimes, an additional study of the relevant topic is recommended. Notice that if there are a number of questions corresponding to the set of conditional components of the topic, these questions are supposed to have the same weight. Only one of them is chosen randomly for the testing.

Since the evaluation of test results is produced for each traversed node of the test tree, the total assessment is gradually accumulated and, if necessary, the learning can be interrupted at any level, with further continuation from the point of interruption.

The proposed technology assumes that both the intermediate and final assessments are available for a group of respondents. Comparison of successive steps of learning by using the proposed technology allows studying the learning process in progress for each of the students. Moreover, it allows designing the learning trajectory for each of them. Interactive comparison of individual results at each learning stage improves the students' mutual relations and supports the healthy competition between the students. It affects positively the internal micro-clime within the group and motivates students in their study.

The final assessment can be both quantitative and qualitative. In order to provide this option, correspondence between qualitative and quantitative scales has been defined.

An important advantage of MDT is the ability to use its intermediary and final testing results for cognitive studies. Notice, that students' educational achievements can be visualized by using various diagrams illustrating the MDT three in dynamics.

\section{Conclusion}

We have introduced the concept of a mixed diagnostic test (MDT), which comprises both unconditional and conditional components. We have also presented a technique for development and implementation of the MDT, being an element of our technology of teaching-testing systems. We have studied the potential of the mixed diagnostic tests in blended learning. Methods of fuzzy logic and threshold functions are used for the assessment based on the MDT.

The use of MDT allows effectively managing curriculum development by constructing individual learning paths (trajectories). It reduces the time and the cost of organization and management of the educational process. Due to the fact that MDT is actually able to replace a teacher in his/her role as a consultant, it is possible to use MDT for blended learning. In our paper, the technique is illustrated by 
the example of the course "Information Technology".

We plan to apply the methodology for development of MDT for a number of other courses having a different structure. We suppose that our approach might be efficient in various mathematical courses, for example, in the course "Discrete Mathematics". We also suppose that the students' discussions concerning the MDT results may increase their motivation for learning and, as a result, the level of their resulting competence. Our future research plans are connected with the high cognitive potential of the MDT visualization at each stage of the learning process. We plan to study regularities of the MDT based education, for example patterns of students' behavior, as well as the process of making pedagogical decisions based on the MDT results. We believe that the above research directions will improve efficiency of the educational process.

\section{Acknowledgements}

Supported by Russian Foundation for Basic Research, projects no. 13-07-98037, 13-07-00373, 14-0700673 and by Russian Humanitarian Foundation project no. 13-06-00709.

\section{References}

Ausburn, L.J. (2004). Course Design Elements Most Valued by Adult Learners in Blended Online Education Environments: An American perspective. Educational Media International, 41(4), 327-337.

Bliuc, A.-M., Goodyear, P., and Ellis, R.A. (2007). Research Focus and Methodological Choices in Studies into Students' Experiences of Blended Learning in Higher Education. Internet and Higher Education, 10, 231-244.

Brusilovsky, P., Knapp, J., and Gamper, J. (2006). Supporting Teachers as Content Authors in Intelligent Educational Systems. International Journal of Knowledge and Learning, 2(3/4), 191-215.

Graham, C. (2006). Blended Learning Systems: Definition, Current Trends, Future Directions. In C. J. Bonk, \& C. Graham (Ed.), The handbook of blended learning: Global perspectives, local designs (pp. 3-21). San Francisco, CA: Pfeiffer Publishing.

Levin I., Kojukhov A. (2013) Personalization of Learning Environments in a Postindustrial Class. Social Media in Higher Education: Teaching in Web 2.0. Editors: M.Patrut and B. Patrut, IGI Global, USA. 105-123.

Levin, I., Kolberg, E., \& Reich, Y. (2004). Robot control teaching with a state machine-based design method. International Journal of Engineering Education, 20(2), 234-243.

Levin, I., Levit, V. (1998). Controlware for Learning with Mobile Robots. Computer Science Education, 8 (3), 181-196.

Singera, F.M., Stoicescu, D. (2011). Using Blended Learning as a Tool to Strengthen Teaching Competences. Procedia Computer Science, 3, 1527-1531.

Varshavsky, V., Marakhovsky, V., Levin, I., \& Kravchenko, N. (2003). Summing Amplifier as a Multi-Valued Logical Element for Fuzzy Control. WSEAS Transactions on Circuit and Systems, (3), 625-631.

Uskov V., Uskov A., (2010). Welcome Address: Computers and Advanced Technology in Education - Perspectives for 20102015. Proceedings of the 13th IASTED International Conference on Computers and Advanced Technology in Education (CATE 2010).

Yankovskaya, A. (1996). Design of Optimal Mixed Diagnostic Test With Reference to the Problems of Evolutionary Computation. Proceedings of the First International Conference on Evolutionary Computation and Its Applications, 292297.

Yankovskaya, A., Yevtushenko, N. (1997). Finite State Machine (FSM) - Based Knowledge Representation in a Computer Tutoring System (pp. 67-74). In New Media and Telematic Technologies for Education in Eastern European Countries, Ed. by P. Kommers \& etc. Twenty University Press Enshede.

Yankovskaya, A.E. (2011). Mixed Diagnostic Tests are a New Paradigm of Construction of Intelligent Learning and Training Systems. Proceedings of the New quality of education in the new conditions, 1, 195-203 (in Russian).

Yankovskaya, A.E., Semenov, M.E. (2009). Construction of the Mixed Tests of the Quality System of Education. Proceedings of the International Scientific Conference (Modern IT\& (e-) Training), Astrakhan, Russia, 125-129 (in Russian).

Yankovskaya, A.E., Semenov, M.E. (2010). Foundation of the Construction of Mixed Diagnostic Tests in Systems for Quality Control of Education. Proceedings of the 13th IASTED International Conference Computers and Advanced Technology in Education (CATE 2010), 142-145.

Yankovskaya, A.E., Semenov, M.E. (2011). Intelligent System for Knowledge Estimation on the Base of Mixed Diagnostic Tests and Elements of Fuzzy Logic. Proceedings of the IASTED International Conference on Technology for Education (TE 2011), 108-113.

Yankovskaya, A.E., Semenov, M.E. (2013). Application Mixed Diagnostic Tests in Blended Education and Training. Proceedings of the IASTED International Conference Web-based Education (WBE 2013), 935-939.

Yankovskaya, A.E., Fuks, I.L., Dementyev, Y.N. (2013). Mixed Diagnostic Tests in Construction Technology of the Training and Testing Systems. International Journal of Engineering and Innovative Technology, 3(5), 169-174. 